\title{
Generalized Warburg impedance on realistic self-affine fractals: Comparative study of statistically corrugated and isotropic roughness ${ }^{\dagger}$
}

\author{
RAJESH KUMAR and RAMA KANT* \\ Department of Chemistry, University of Delhi, Delhi 110007 \\ e-mail: rkant $@$ chemistry.du.ac.in
}

\begin{abstract}
We analyse the problem of impedance for a diffusion controlled charge transfer process across an irregular interface. These interfacial irregularities are characterized as two class of random fractals: (i) a statistically isotropic self-affine fractals and (ii) a statistically corrugated self-affine fractals. The information about the realistic fractal surface roughness has been introduced through the bandlimited power-law power spectrum over limited wave numbers. The details of power spectrum of such roughness can be characterized in term of four fractal morphological parameters, viz. fractal dimension $\left(D_{H}\right)$, lower $(\ell)$, and upper $(L)$ cut-off length scales of fractality, and the proportionality factor $(\mu)$ of power spectrum. Theoretical results are analysed for the impedance of such rough electrode as well as the effect of statistical symmetries of roughness. Impedance response for irregular interface is simplified through expansion over intermediate frequencies. This intermediate frequency expansion with sufficient number of terms offers a good approximation over all frequency regimes. The Nyquist plots of impedance show the strong dependency mainly on three surface morphological parameters i.e. $D_{H}, \ell$ and $\mu$. We can say that our theoretical results also provide an alternative explanation for the exponent in intermediate frequency power-law form.
\end{abstract}

Keywords. Warburg impedance; rough electrode; realistic self-affine fractals; diffusion controlled.

\section{Introduction}

Electrochemical impedance spectroscopy is a powerful technique for investigating electrochemical systems and processes. The main strength lies in its ability to interrogate relaxation phenomenon. In contrast to other electrochemical techniques, the electrochemical impedance spectroscopy is noninvasive and can be used for investigating bulk as well as interfacial processes. The method of impedance measurement is used extensively in applied systems like: corrosion, ${ }^{1}$ bio-sensors, ${ }^{2}$ batteries, ${ }^{3,4}$ characterization of thin film and coating, ${ }^{5,6}$ electrochemical machining, ${ }^{7}$ fuel cell, ${ }^{8}$ bio-electrochemistry, ${ }^{9}$ etc. and also to study the mechanism of interfacial processes, electrode kinetics, double layer studies, evaluation of parameters like rate constants and double layer capacitance in electrochemistry. ${ }^{10-12}$ The problem of diffusive transport to and across an irregular interface is common and are of general interest. Diffusion-limited processes on such interfaces show anomalous diffusive behaviour and realized in various physical phenomena such as: spin

\footnotetext{
${ }^{\dagger}$ Dedicated to the memory of the late Professor S K Rangarajan *For correspondence
}

relaxation, ${ }^{13}$ fluorescence quenching ${ }_{,}^{13,14}$ heterogeneous catalysis, ${ }^{15}$ enzyme kinetics, ${ }^{16}$ heat diffusion, ${ }^{17}$ membrane transport, ${ }^{18,19}$ electrochemistry, ${ }^{20-44}$ and impedance response. ${ }^{24-26,40-46}$

Most of the real interfacial system can exhibit complex shapes with varying degree of irregularity or disorder. Such complex unconventional geometrical presence influences the electrode response. The recent interest in realistic fractal geometry ${ }^{26,28,29,40,44}$ has helped to understand the influence of surface disorder over the electrode response. The surface irregularities over realistic objects are characterized as two class of random fractals: (i) statistically isotropic fractals and (ii) statistically corrugated fractals. Theoretical understanding in electrochemistry for these surfaces offers solution to several open ended questions in this area. Particularly, our focus in this communication is on realistic surface fractals with limited length scale of irregularities which are frequently looked upon as self-affine fractals (figure 1). ${ }^{26-29,44,47}$

Diffusion-limited electrode reactions are frequently observed in electrode kinetics. The total admittance, $Y(\omega)$, of an interfacial redox reaction, $O+n e^{-} \rightleftharpoons R$, driven by sinusoidal interfacial poten- 
tial $\left(\delta \eta=E-E_{e}=\eta_{0} e^{i \omega t}\right.$, where $\delta \eta$ is the magnitude of overpotential, $E_{e}$ is the equilibrium potential and $\eta_{0}$ is the magnitude of applied sinusoidal potential, $i=\sqrt{-1}, \omega$ is angular frequency and $t$ is time) can be obtained by solving appropriate diffusion equation.

$$
\frac{\partial C_{\alpha}(\vec{r}, t)}{\partial t}=D_{\alpha} \nabla^{2} C_{\alpha}(\vec{r}, t),
$$

where $\alpha \equiv O, R$, represents the oxidized or reduced species, $C_{\alpha}(\vec{r}, t)$ is the concentration of $\alpha$ th species, $D_{\alpha}$ is the bulk diffusion constant of $\alpha$ th species, and $\vec{r}$ is the three-dimensional vector; $\vec{r} \equiv(x, y, z)$. The concentration profile of the form $\delta C_{\alpha}(\vec{r}) e^{i \omega t}$ satisfies the diffusion equation and can be expressed as:

$$
i \omega \delta C_{\alpha}(\vec{r})=D_{\alpha} \nabla^{2} \delta C_{\alpha}(\vec{r}),
$$

where $\delta C_{\alpha}(\vec{r})$ is the difference between surface and bulk concentration, $i=\sqrt{-1}$ and $\omega$ is the angular frequency. There is a local transfer kinetics limita-

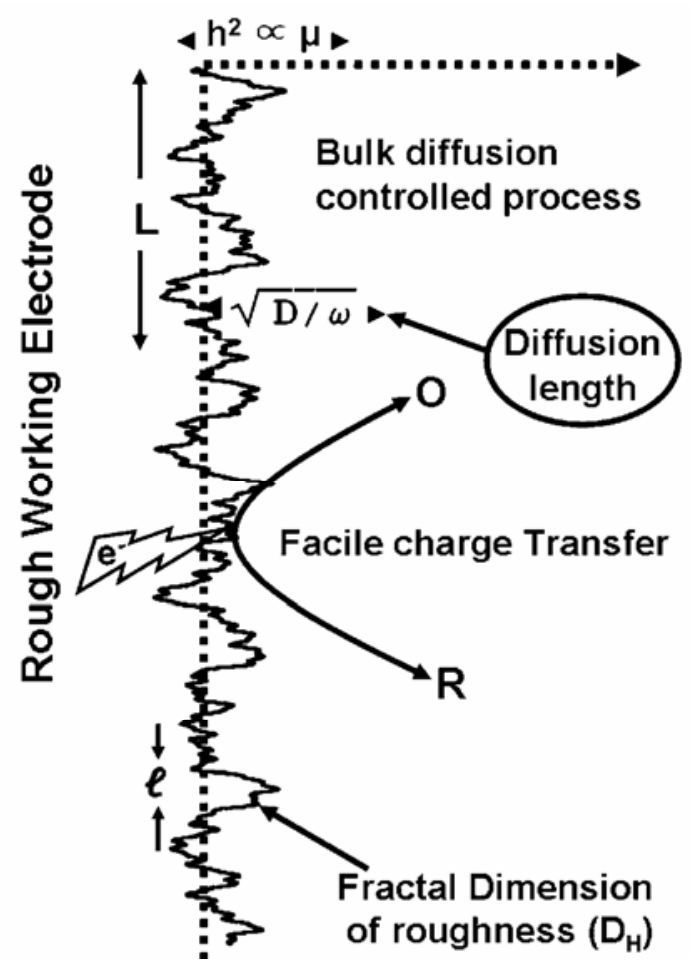

Figure 1. Schematic diagram of a rough working electrode, where a charge transfer reaction $O(\mathrm{Sol})+n e^{-}$ $\rightleftharpoons R(\mathrm{Sol})$ under Nernstian condition and bulk diffusion of redox species is taking place. Various length scales, which control the response of electrode/electrolyte interface are smallest $(\ell)$ and largest $(L)$ length scale of roughness, fractal dimension $\left(D_{H}\right)$, mean square width $\left(h^{2} \propto \mu\right)$ of interface and the diffusion length $\sqrt{D / \omega}$. tion at the interface $(\zeta)$ which can be obtained under Nernstian boundary constraint (for simplicity we assume $D_{O}=D_{R}=D$ ). Hence, the linearized Nernstian condition at the interface can be expressed as:

$$
\delta C_{S}(z=\zeta(x, z))=-n f \eta_{0}\left(\frac{1}{C_{O}^{0}}+\frac{1}{C_{R}^{0}}\right),
$$

where $\delta C_{S}(z=\zeta(x, y))$ is the surface concentration, $n$ being the number of electron transferred, $f=F / R T$ (where $F$ is Faraday constant, $R$ is gas constant and $T$ being the temperature). $\eta_{0}$ is the magnitude of applied sinusoidal potential. $C_{O}^{0}$ and $C_{R}^{0}$ are the bulk concentrations of oxidized and reduced species, respectively. Here at initial time and far off from the interface, we assume uniform initial and bulk concentration $C_{\alpha}^{0}$ viz.

$$
C_{\alpha}(\vec{r}, r=0)=C_{\alpha}(z \rightarrow \infty, t)=C_{\alpha}^{0} .
$$

The formalism for diffusion processes on realistic random boundary value problem and its approximate results for sharp cut-off corrugated self-affine fractals is described in reference (26) and formalism for diffusion processes on realistic random boundary value problem and its approximate results for sharp cut-off - band limited power spectrum for isotropic self-affine fractals is described in reference (44). We wish to use these perturbative solutions of boundary value problem on realistic fractals because these are most frequent form of roughness encountered in rough electrodes. In the absence of roughness, admittance for above mentioned problem has well known form which is called Warburg admittance. Warburg admittance of planar electrode ${ }^{45}$ is given as:

$$
Y_{W}(\omega)=A_{0} \Gamma(i \omega D)^{1 / 2},
$$

where $A_{0}$ is the projected area of the surface, $\Gamma$ is specific diffusion capacitance, defined as

$$
\Gamma=n^{2} F^{2} / R T\left(\frac{1}{C_{O}^{0}}+\frac{1}{C_{R}^{0}}\right) .
$$

Warburg admittance inferred that the admittance under diffusion-controlled process is directly proportional to the area of electrode, specific capacitance and the complex depletion layer thickness. 
It is well-known and understood that the analytical approaches to such interfaces are difficult and solved under various degree of approximations. The scaling, numerical and experimental studies ${ }^{20-22,26-29,37,44}$ have shown that the admittance, $Y(\omega)$, in the intermediate frequency domain is expressed as powerlaw relation. The scaling form for admittance is:

$$
Y(\omega) \sim(i \omega)^{\beta},
$$

where the exponent, $\beta$, depends on the interfacial roughness. De Gennes ${ }^{13}$ and Pajkossy ${ }^{23}$ gave less precise explanation for the scaling exponent for intermediate frequency diffusion limited charge transfer process, i.e. $\beta=\left(D_{H}-1\right) / 2$. Later similar results discussed for other diffusion controlled situations such as impedance of rough electrode, ${ }^{20,21}$ current or impedance for the diffusion limited transfer at irregular electrode surface. ${ }^{20-22,26-29,35-37,44}$ These works consider two charge transfer conditions: (i) Nernstian charge transfer ${ }^{26,28,29,44}$ and (ii) Quasi-reversible charge transfer ${ }^{36,37}$ These results were obtained both in terms of an arbitrary roughness deterministic profile and an arbitrary roughness power spectrum for random roughness profile. An important power spectrum for approximately self-affine fractals can have band-limited power-law form. ${ }^{27-29,36,37,44,47}$

Most of the studies on realistic fractals (statistically isotropic band limited and statistically corrugated band limited) address the questions related to roughness with sharp cut-offs. ${ }^{28,44}$ Palasantzas ${ }^{40}$ also used the formalism discussed in us in reference (26) to study the admittance of self-affine rough electrode but their analysis is limited to high frequency region. They completely missed the essential intermediate anomalous frequency behaviour of this problem. Here we use perturbative solution for realistic fractal roughness formulated in reference (26) and reference (44) for corrugated self-affine random fractals and isotropic self-affine fractal, respectively.

In this paper, we analyse the electrochemical impedance response in Nyquist form for statistically corrugated as well as isotropic self-affine fractals. Comparative study between statistically corrugated self-affine fractal and isotropic self-affine fractal differs in qualitative responses which emphasizes the effect of dimensionality.

\section{Statistical description of random surface fractals}

Statistical models are employed here for rough electrodes, and the electrode-electrolyte interface is considered a random surface. Roughness of these surfaces can be modelled as approximately selfaffine fractal roughness having structure factor of the form which is band-limited power-law function $^{27-29,44,47}$ and such surfaces exhibit statistical self-resemblance over limited range of length scales. It is important to emphasize that the structure factor or power spectrum which is related to two-point correlation function and consist equivalent surface morphological information.

The admittance equation for bulk diffusion followed by a fast charge transfer reaction at electrode/electrolyte interface was analysed by KantRangarajan. ${ }^{26}$ The ensemble averaged admittance at the stationary, Gaussian random electrode surface which is related to its structure factor of roughness. The generalized expression is given as:

$$
\begin{aligned}
\langle Y(\omega)\rangle= & Y_{W}(\omega)\left[1+\frac{1}{(2 \pi)^{2}} \sqrt{\frac{i \omega}{D}} \int d^{2} K\right. \\
& \left.\left(\sqrt{K^{2}+\frac{i \omega}{D}}-\sqrt{\frac{i \omega}{D}}\right)\left\langle|\hat{\zeta}(K)|^{2}\right\rangle\right],
\end{aligned}
$$

where $\langle Y(\omega)\rangle$ is the ensemble averaged admittance for the rough electrode, $Y_{W}(\omega)$ is admittance for smooth electrode commonly known as Warburg admittance and expressed by (4). $\left\langle|\hat{\zeta}(K)|^{2}\right\rangle$ is the structure factor or power spectrum of roughness which is function of wavenumber, $K$. The generalized diffusional impedance (Warburg) (6) on arbitrary random rough electrode is proportional to the admittance of smooth electrode (i.e. Warburg admittance) and frequency-dependent complex dynamic roughness factor. This dynamic roughness factor contains all information about the surface geometric disorder through power spectrum of roughness. The description of anomalous diffusion impedance due to random fractals can be studied over two types of fractals namely: (i) statistically corrugated selfaffine fractals and (ii) statistically isotropic selfaffine fractals. The description of these surfaces is discussed in the following subsections.

\subsection{Statistically corrugated self-affine fractals}

The power spectrum can be obtained for disordered surface profile using various scanning techniques. Some of the measured power spectra show constant 
magnitude up to certain wavenumber and exhibit power-law form with high wavenumber. ${ }^{48}$ The power spectrum of roughness, $\left\langle|\hat{\zeta}(K)|^{2}\right\rangle$, for such corrugated self-affine fractal surfaces can be defined in terms of power-law function ${ }^{26,27,47}$ as:

$$
\left\langle|\hat{\zeta}(K)|^{2}\right\rangle=\left\{\begin{array}{ll}
\mu L^{-\left(2 D_{H}-5\right)}, & K<1 / L \\
\mu K^{2 D_{H}-5}, & 1 / L \leq K \leq 1 / \ell \\
0, & K \geq 1 / \ell
\end{array}\right\},
$$

where $K$ is the wavenumber in one-dimension, $D_{H}$ is fractal dimension, which describe the scale invariance property of roughness, $1 / L$ and $1 / \ell$ are related to low wavenumber cut-off and high wavenumber cut-off, respectively. The lower cut-off length $(\ell)$ can be defined as the length above which surface shows fractal behaviour and upper cut-off length scale $(L)$ is the length below which surface exhibit fractal behaviour. $\mu$ is the proportionality factor which is related to the topothesy of fractals. ${ }^{47}$ Topothesy $\tau$ is defined as the horizontal distance between two points on which structure factor, equal to $\tau^{2}$, can be expressed as: $:^{47}$

$$
\left\langle\left[\zeta(x, y)-\zeta\left(x^{\prime}, y^{\prime}\right]^{2}\right\rangle=\tau^{d+2-\alpha} X^{\alpha-d},\right.
$$

where $\zeta(x, y)$ is the centered surface profile function, the angular brackets designate ensemble averaging and distance as

$$
X=\left[\left(x-x^{\prime}\right)^{2}+\left(y-y^{\prime}\right)^{2}\right]^{1 / 2},
$$

$d$ is dimension and $\alpha$ is the spectral exponent and restricted within $d<\alpha<d+2$, in order to show that surface profile is self-affine (in statistical sense). Topothesy of an 'ideal' fractal surface is related to the normalizing factor (strength) of the power-law spectra $(\mu)$ by the following relation:

$$
\tau^{d+2-\alpha}=\frac{\mu \pi^{d / 2} 2^{d+2-\alpha} \Gamma((d+2-\alpha) / 2)}{(\alpha-d) \Gamma(\alpha / 2)}
$$

for the cases $d=1$ (corrugated) and $d=2$ (isotropic), topothesy expression can be simplified. Power spectrum, $\left\langle|\hat{\zeta}(K)|^{2}\right\rangle$, has unit of $\mathrm{cm}^{3}$ for corrugated self-affine fractals and $\mathrm{cm}^{4}$ for isotropic self-affine fractals. $\mu$ has unit of $\mathrm{cm}^{2 D_{H}-2}$ for corrugated self-affine fractals and $\mathrm{cm}^{2 D_{H}-3}$ for self-affine isotropic fractals, if $\mu \rightarrow 0$ means there is no rough- ness and under this limit the whole generalized admittance expression equals to the plane electrode response. For corrugated self-affine fractal surfaces integral

$$
\int_{-\infty}^{\infty} d^{2} K(.)=\int_{-\infty}^{\infty} d K_{x} \int_{-\infty}^{\infty} d K_{y}(.)
$$

in (6) can be expressed as

$$
\int_{-\infty}^{\infty} d K_{x}(.)=2 \int_{0}^{\infty} d K_{x}(.)
$$

Substituting (7) in (6) and solving resultant integral, we obtain the ensemble averaged admittance expression for statistically corrugated band limited fractal surfaces and can be expressed as:

$$
\begin{aligned}
& \left\langle Y_{\text {Corr }}(\omega)\right\rangle=Y_{W}(\omega)\left[1+R_{L}^{c}(\omega)+R_{I}^{c}(\omega)\right], \\
& R_{L}^{c}(\omega)=-\frac{i \omega}{D} \frac{\mu L^{-\left(2 \delta_{c}-1\right)}}{4 \pi} \\
& {\left[4+L \sqrt{\frac{i \omega}{D}}\left(\log \left(\frac{i \omega}{D\left(\frac{1}{L}+\sqrt{\frac{1}{L^{2}}+\frac{i \omega}{D}}\right)^{2}}\right)\right)\right.} \\
& -2 \sqrt{\frac{D}{i \omega} \sqrt{\frac{1}{L^{2}}+\frac{i \omega}{D}}} \\
& R_{I}^{c}(\omega)=\frac{i \omega}{D}\left[\frac{\mu L^{-\left(2 \delta_{c}-1\right)}}{\pi\left(2 \delta_{c}-1\right)}-\frac{\mu \ell^{-\left(2 \delta_{c}-1\right)}}{\pi\left(2 \delta_{c}-1\right)}+\psi^{c}(\omega)\right] \\
& \psi^{c}(\omega)=\frac{\mu \ell^{-\left(2 \delta_{c}-1\right)}}{\pi\left(2 \delta_{c}-1\right)}{ }_{2} F_{1}\left[\delta_{c}-\frac{1}{2}, \frac{-1}{2}, \delta_{c}+\frac{1}{2}, \frac{i D}{\ell^{2} \omega}\right] \\
& -\frac{\mu L^{-\left(2 \delta_{c}-1\right)}}{\pi\left(2 \delta_{c}-1\right)}{ }_{2} F_{1}\left[\left[\delta_{c}-\frac{1}{2}, \frac{-1}{2}, \delta_{c}+\frac{1}{2}, \frac{i D}{L^{2} \omega}\right]\right],
\end{aligned}
$$

here $\delta_{c}=\left(D_{H}-3 / 2\right)$ is deviation from the Brownian fractal dimension for corrugated surfaces and ${ }_{2} F_{1}$ is 
the hypergeometric function. ${ }^{49} R_{L}^{c}(\omega)$ is the low wave number contribution in roughness (from flat region of wave number) and $R_{I}^{c}(\omega)$ is the intermediate wave number contribution in roughness for corrugated self-affine fractal surfaces. The admittance expression (8) is dependent on four fractal morphological parameters of power spectrum of roughness and phenomenological diffusion length. The anomalous admittance region for the intermediate frequency can be expressed in terms of series expansion of two hypergeometric functions shown in (8) viz. one for small $\left(D / L^{2} \omega\right)$ and another for large $\left(D / \ell^{2} \omega\right)$ and retaining only the leading orders. The admittance equation for the intermediate anomalous region for statistically corrugated fractal surfaces, first five leading terms can be written as:

$$
\left.\begin{array}{c}
\left\langle Y_{\text {Corr }}(\omega)\right\rangle \approx Y_{W}(\omega) \\
1+\frac{i \omega}{d}\left(\begin{array}{c}
-\frac{\mu \ell^{-\left(2 \delta_{c}-1\right)}}{\pi\left(2 \delta_{c}-1\right)}-\frac{\mu}{2 \pi^{3 / 2}\left(2 \delta_{c}-1\right)} \\
\Gamma\left(\delta_{c}+1 / 2\right) \Gamma\left(-\delta_{c}\right)\left(\frac{i \omega}{d}\right)^{\delta_{c}-1 / 2} \\
+\frac{\mu \ell^{-2 \delta_{c}}}{2 \pi\left(\delta_{c}\right)} \sqrt{\frac{d}{i \omega}}+\frac{\mu \ell^{-2\left(\delta_{c}-1\right)}}{4 \pi\left(\delta_{c}-1\right)} \\
\sqrt{\frac{i \omega}{d}}+\frac{\mu \ell^{2-2\left(\delta_{c}-1\right)}}{16 \pi\left(2-\delta_{c}\right)}\left(\frac{i \omega}{d}\right)^{3 / 2}
\end{array}\right)
\end{array}\right]
$$

here $\Gamma($.$) , is Gamma function and \delta_{c}=\left(D_{H}-3 / 2\right)$. Equation (9) is obtained for intermediate frequency regime of admittance for corrugated random fractals. Equation (9) offers a good approximation of equation (8) in all frequency regimes (see (23) in reference 26 represent the special case of this equation).

\subsection{Statistically isotropic self-affine fractals}

The power spectrum of roughness, $\left\langle|\hat{\zeta}(K)|^{2}\right\rangle$ for isotropic self-affine fractal can be expressed in terms of power-law function ${ }^{28,29,37,44,47}$ as:

$$
\left\langle\left|\hat{\zeta}\left(\vec{K}_{\|}\right)\right|^{2}\right\rangle=\left\{\begin{array}{ll}
\mu L^{-2 D_{H}-7}, & \left|\vec{K}_{\|}\right|<1 / L \\
\mu\left(\left|\vec{K}_{\|}\right|\right)^{2 D_{H}-7}, & 1 / L \leq\left|\vec{K}_{\|}\right| \leq 1 / \ell \\
0, & \left|\vec{K}_{\|}\right| \geq 1 / \ell
\end{array}\right\},
$$

here $\vec{K}_{\|}$is a wave vector in two-dimension. $\mu$ is the proportionality factor known as strength of fractality, which is related to the topothesy of fractals. ${ }^{47}$ $\ell$ is the lower cut-off length and $L$ is the upper cutoff length. The angular brackets denote an ensemble average over various possible surface configurations. For isotropic self-affine fractals, $\int d^{2} K$ in the integrand represents a double integral over two components of wave-vector with limits $-\infty$ to $\infty$ and for statistically isotropic surface this simplifies to:

$$
\int d^{2} K=2 \pi \int_{0}^{\infty} \mathrm{d} K K
$$

Substituting (10) in (6) and solving resultant integral, we have ensemble averaged admittance expression for a band-limited isotropic fractal and can be expressed as:

$$
\begin{aligned}
&\left\langle Y_{I s o}(\omega)\right\rangle=Y_{W}(\omega)\left[1+R_{L}(\omega)+R_{I}(\omega)\right] \\
& R_{L}(\omega)=-\frac{i \omega}{D} \frac{\mu L^{-2 \delta}}{4 \pi} {\left[1+\frac{2 i \omega}{3 D} L^{2}-\frac{2}{3 L} \sqrt{\frac{D}{i \omega}}\left(1+\frac{i \omega}{D} L^{2}\right)^{3 / 2}\right] } \\
& R_{I}(\omega)=\frac{i \omega}{D}\left[\frac{\mu L^{-2 \delta}}{4 \pi \delta}-\frac{\mu \ell^{-2 \delta}}{4 \pi \delta}+\psi(\omega)\right] \\
& \psi(\omega)=\frac{\mu \ell^{-2 \delta}}{4 \pi \delta}{ }_{2} F_{1}\left[\delta, \frac{-1}{2}, \delta+1, \frac{i D}{\ell^{2} \omega}\right] \\
&-\frac{\mu \ell^{-2 \delta}}{4 \pi \delta}{ }_{2} F_{1}\left[\delta, \frac{-1}{2}, \delta+1, \frac{i D}{L^{2} \omega}\right],
\end{aligned}
$$

here $\delta=\left(D_{H}-5 / 2\right)$ is deviation from the Brownian fractal dimension for isotropic surfaces and ${ }_{2} F_{1}$ is the hypergeometric function. ${ }^{49}$ The function, $R_{L}(\omega)$, is the low wave number contribution of roughness and $R_{I}(\omega)$, is the intermediate wave number contribution of roughness. This admittance expression is dependent on four fractal morphological parameters of power spectrum of roughness and phenomenological diffusion length. The generalization of this result is obtained in reference 44 for sharp cut-off 
spectrum. The anomalous admittance intermediate frequency region expansion of two hypergeometric functions in (11) can be expressed as: (see appendix in reference (44). Note that contribution from $R_{L}(\omega)$ is insignificant in intermediate frequency regime)

$$
\left\langle Y_{I S O}(\omega)\right\rangle \approx Y_{W}(\omega)
$$

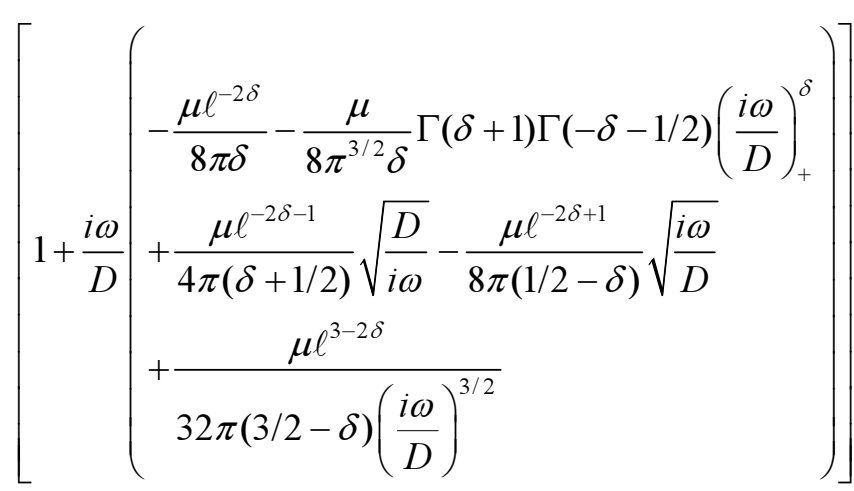

here $Y_{W}(\omega)$ is the Warburg admittance and $\Gamma($.$) , is$ Gamma function. ${ }^{49}$ This simplified expression is suitable to obtain intermediate frequency dependence of anomalous admittance. Admittance expression in intermediate frequency region is a dominant function of the three fractal morphological parameters say $\left(D_{H}, \ell, \mu\right)$.

Equations $(8,9,11,12)$ generalizes the conventional representation of the Warburg admittance on smooth surface electrode to the fractally rough electrode where roughness is characterized by its power spectrum. These equations generalize Warburg admittance response for a realistic statistically corrugated and statistically isotropic self-affine fractal roughness. We will analyse Nyquist plots of these equations in the following section.

\section{Results and discussion}

The general aspect for the diffusion limited admittance/impedance response on realistic fractal electrode is depicted through the Nyquist diagram. Here, we analyse graphically how the electrochemical impedance response; under bulk diffusion and facile interfacial charge transfer condition; is influenced by the surface morphological features and how they deviate from smooth geometry response. These figures show clearly that the extent of roughness has an important effect on impedance response. Usually, Nyquist plots represent the real component of impedance $\left(Z^{\prime}(\omega)\right)$ along $x$-axis and imaginary component of impedance $\left(-Z^{\prime \prime}(\omega)\right)$ along $y$-axis with varying frequency. The higher frequency region is observed towards left and lower frequency region is observed towards right in the plots. The assumption of semi-infinite diffusion on smooth surface made by Warburg for the derivation of diffusional impedance signifies that the real component of impedance $\left(Z^{\prime}(\omega)\right)$ and imaginary component of impedance $\left(-Z^{\prime \prime}(\omega)\right)$ have the same value and hence has a straight line appearance with a phase angle of $45^{\circ}$, but presence of roughness violates this assumption.

Logarithmic Nyquist plot is shown in figure 2 to emphasize various regimes in frequency domain. This figure also emphasizes the deviation and distinguishing features of diffusional impedance response from the smooth electrode response. Various curves are plotted under the variation of fractal dimension $\left(D_{H}\right)$ while all other parameters are kept fixed. The real component of impedance $\left(Z^{\prime}(\omega)\right)$ and imaginary component of impedance $\left(-Z^{\prime \prime}(\omega)\right)$ of

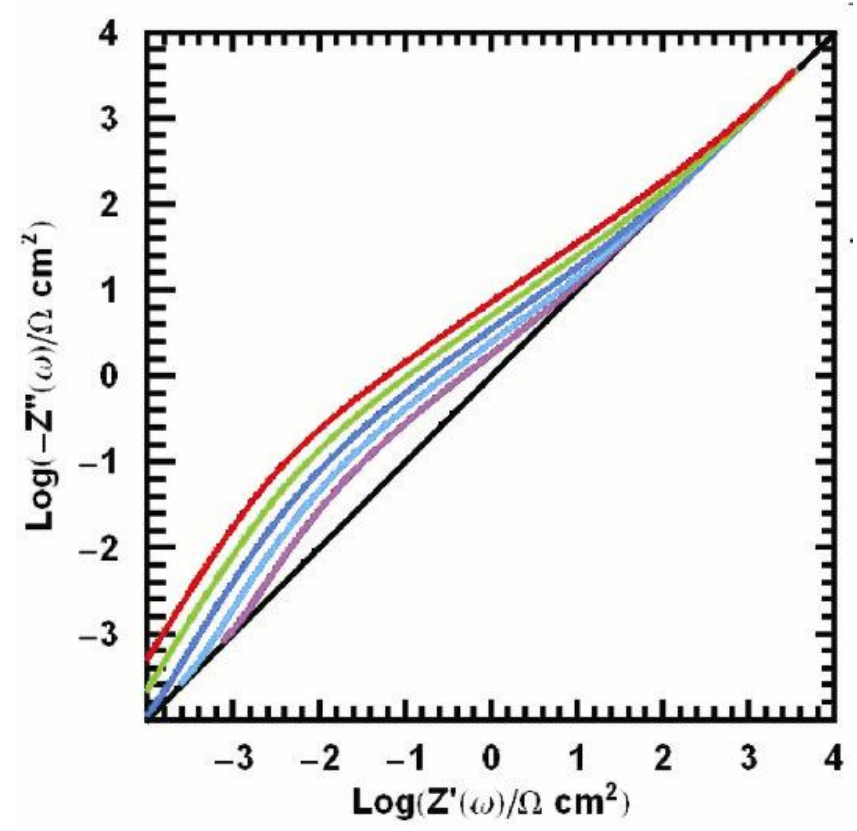

Figure 2. Logarithmic Nyquist plots for isotropic selfaffine fractals showing effect of fractal dimension on the diffusion impedance. Plot is generated for fixed, $\ell=40 \mathrm{~nm}, \mu=2 \times 10^{-6}$ (a.u.) and $L=4 \mu \mathrm{m}$, while varying $D_{H}$ as $2 \cdot 25,2 \cdot 3,2 \cdot 35,2 \cdot 4,2 \cdot 45$, diffusion coefficient $D=5 \times 10^{-6} \mathrm{~cm}^{2} / \mathrm{s}$, frequency $\omega\left(\mathrm{s}^{-1}\right)=10^{-3}$ to $10^{3}$ and concentration $C_{\mathrm{O}}^{0}=C_{\mathrm{R}}^{0}=15 \mathrm{mM}$ are used. 
Nyquist plots are related with magnitude of impedance and phase angle. Therefore, any change in real component and imaginary component of impedance will affect the magnitude of impedance and phase angle response. ${ }^{44}$ The logarithmic plots of components of impedance in this figure merges with the planar response (lowest curve) at very low and very high frequency. Deviation from planar response corresponds to crossover frequencies i.e., outer crossover frequency $\left(\omega_{\mathrm{O}} \sim D / L^{2}\right)$ and inner crossover frequency $\left(\omega_{i} \sim D / \ell^{2}\right)$, which emphasize the effect of roughness. Outer crossover frequency $\left(\omega_{\mathrm{O}} \sim D / L^{2}\right)$ is related to upper length scale cut-off observed at low frequency where $Z^{\prime}(\omega) \cong-Z^{\prime \prime}\left(\omega_{0}\right)$ and inner crossover frequency cut-off $\left(\omega_{i} \sim D / \ell^{2}\right)$ is related to lower length scale cut-off corresponding to first inflexion point on left hand side of curves and is observed at high frequency. The anomalous powerlaw frequency dependence of impedance Bode plot ${ }^{44}$ can be seen above the inner crossover frequency and below the outer crossover frequency which is intermediate frequency region. The fractal dimension $\left(D_{H}\right)$ increases from bottom to top in this figure and magnitude of $-Z^{\prime \prime}(\omega)$ increases with $D_{H}$ for a given value of $Z^{\prime}(\omega)$.

Traditionally, the Nyquist plots have been found in non-logarithmic scale. We have plotted conventional Nyquist plots in figure 3 showing the influence of various fractal roughness features and difference is emphasized over the planar surface response.

Figure 3(a) shows the Nyquist plots for diffusional impedance (Warburg) response under the variation of fractal dimension $\left(D_{H}\right)$ and the response of smooth (planar) electrode is shown in lowest curve. The fractal dimension $\left(D_{H}\right)$ increases from bottom to top in this figure while the other morphological parameters of power spectrum are fixed. These are lower cut-off length $(\ell=40 \mathrm{~nm})$, strength of fractality $\left(\mu=2 \times 10^{-6}\right.$ (arbitrary units)) and upper length scale cut-off $(L=4 \mu \mathrm{m})$. In these plots frequency varies from $10^{-2}$ to $10^{2}$ which correspond mainly to the intermediate frequency region. The real component of impedance $\left(Z^{\prime}(\omega)\right)$ and imaginary component of impedance $\left(-Z^{\prime \prime}(\omega)\right)$ merges with the planar response of Warburg impedance at higher frequency. The signature of anomalous power-law frequency dependence of impedance can be seen in approximately linear region. The magnitude of imaginary component of impedance $\left(Z^{\prime \prime}(\omega)\right)$ increases as the fractal dimension increases for a fixed value of $Z^{\prime}(\omega)$.
Figure 3(d) shows the influence of fractal dimension $\left(D_{H}\right)$ over the diffusional impedance response of statistically corrugated fractal electrode. The fractal dimension $\left(D_{H}\right)$ increases from bottom to top in this figure while other surface morphological parameters kept constant. These are lower cut-off length $(\ell=40 \mathrm{~nm})$, strength of fractality $\left(\mu=15 \times 10^{-2}\right.$ (arbitrary units)) and upper length scale cut-off $(L=4 \mu \mathrm{m})$. In this case the strength of fractality differs in magnitude as compared to isotropic self-affine fractals, because the electrochemical impedance response for statistically corrugated fractal surfaces is very low as compared to the isotropic self-affine fractals with equivalent set of roughness parameters. Therefore, we increase the value of strength of fractality (which is related to the width of interface) for statistically corrugated fractal surface, so that we can get the same qualitative response as we get in case of isotropic selfaffine fractals. The approximate power-law can be seen in approximately linear region. Here also the magnitude of imaginary component of impedance increases with increase in fractal dimension.

In figure 3(b) we observe the influence of lower cut-off length $(\ell)$ scale over the electrochemical impedance response. From this plot one can say that the intermediate frequency admittance scaling exponent, $\beta$, is usually related in less precise calculation to fractal dimension $\left(D_{H}\right)$. There are some other surface morphological parameters on which the electrochemical response depends. These parameters are lower length cut-off $(\ell)$ and strength of fractality $(\mu)$. Parameters which are fixed for this figure are fractal dimension $\left(D_{H}=2.4\right)$, strength of fractality $\left(\mu=2 \times 10^{-6}\right.$ (arbitrary units)) and upper length scale cut-off $(L=4 \mu \mathrm{m})$ with varying $\ell$ which is increases from top to bottom. In this plot we observe that the magnitude of imaginary component of impedance $\left(Z^{\prime \prime}(\omega)\right)$ decreases with increase in lower cut-off length scale. This plot exhibits that, for low roughness surface, the impedance behaviour follows the same pattern as for planar surface, but as the roughness increases due to decrease in lower cut-off length value, there is increase in imaginary part of impedance for a given value of real part of impedance. The approximate linear behaviour in intermediate frequency region corresponds to identical functional dependence of real and imaginary components. All curves merge with a planar response curve at higher frequency. 

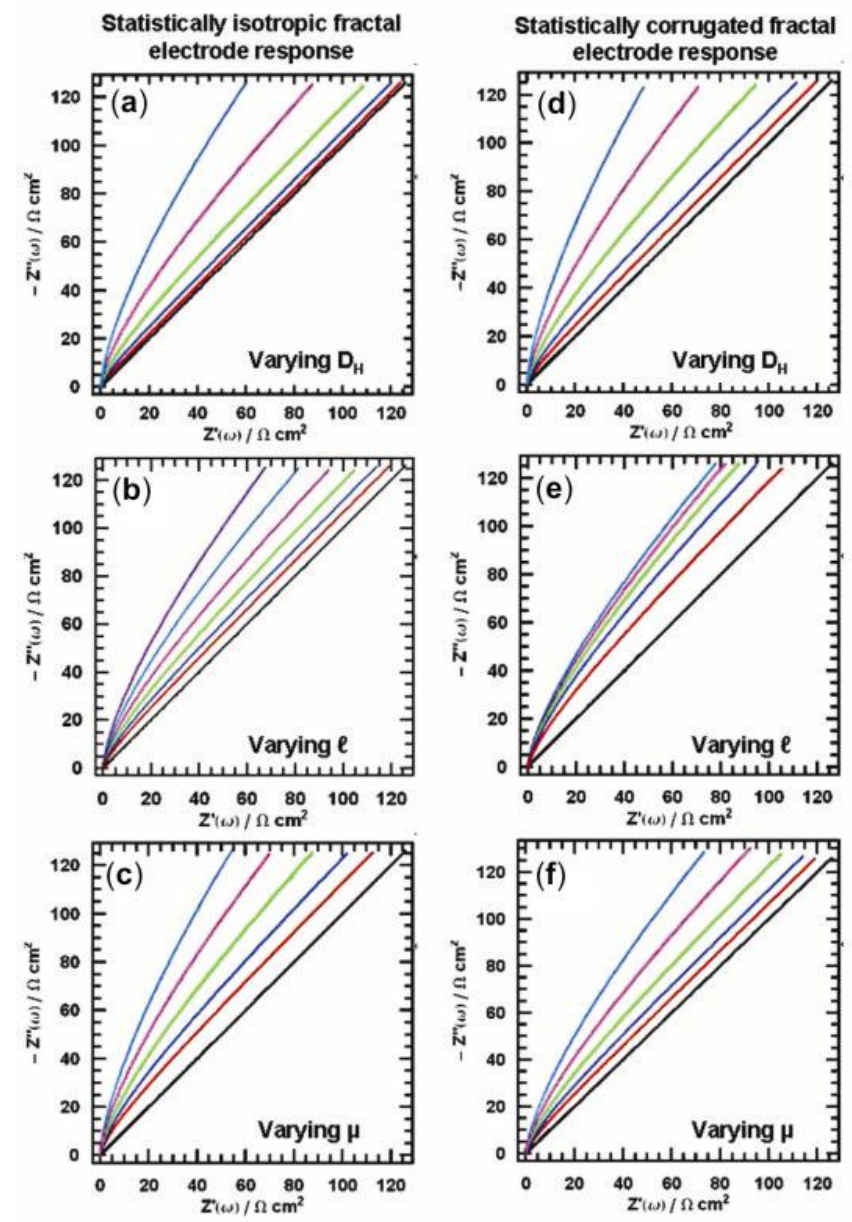

Figure 3. First column of the graphs represent Nyquist plots for isotropic self-affine fractals: (a) Effect of fractal dimension on the diffusion impedance. Plot is generated for fixed, $\ell=40 \mathrm{~nm}, \mu=2 \times 10^{-6}$ (a.u.) and $L=4 \mu \mathrm{m}$, while varying $D_{H}$ as $2 \cdot 25,2 \cdot 3,2 \cdot 35,2 \cdot 4,2 \cdot 45$, diffusion coefficient $D=5 \times 10^{-6} \mathrm{~cm}^{2} / \mathrm{s}$, frequency $\omega\left(\mathrm{s}^{-1}\right)=10^{-2}$ to $10^{2}$ and concentration $C_{\mathrm{O}}^{0}=C_{\mathrm{R}}^{0}=15 \mathrm{mM}$ are used. (b) Effect of lower length scale cut-off. Plot is generated for fixed $D_{H}=2.4, \mu=2 \times 10^{-6}$ (a.u.) and $L=4 \mu \mathrm{m}$, while varying $\ell(\mathrm{nm})$ as $15,30,60,120,240,480$, other parameters are fixed as in figure 3(a). (c) Effect of strength of fractality. Plot is generated for fixed $\ell=40 \mathrm{~nm}, L=$ $4 \mu \mathrm{m}, D_{H}=2.4$ while varying $\mu\left(10^{-6}\right.$ a.u. $)$ as $0.5,1 \cdot 0$, $2 \cdot 0,4 \cdot 0,8 \cdot 0$. Second column of the graphs represent $\mathrm{Ny}-$ quist plots for corrugated random self-affine fractals: (d) Effect of fractal dimension, parameters are $\ell=40 \mathrm{~nm}$, $\mu=15 \times 10^{-2}$ (a.u.) and $L=4 \mu \mathrm{m}$, while varying $D_{H}$ as $1.25,1.3,1.35,1.4,1.45$, and other parameters are same as in other plots. (e) Effect of lower length cut-off. Parameters are $D_{H}=1.4, \quad \mu=15 \times 10^{-2}$ (a.u.) and $L=$ $4 \mu m$, while varying $\ell(\mathrm{nm})$ as $125,250,500,1000,2000$. (f) Effect of strength of fractality is plotted where roughness parameters are $\ell=40 \mathrm{~nm}, L=4 \mu \mathrm{m}, D_{H}=1.4$ while varying $\mu\left(10^{-2}\right.$ a.u. $)$ as $1 \cdot 0,2 \cdot 0,4 \cdot 0,8 \cdot 0,16 \cdot 0$.

In figure 3(e), we understand how the lower cutoff length $(\ell)$ value influences the diffusional impedance response for statistically corrugated fractal surfaces and how they differ from the smooth geometry response. Lower cut-off length $(\ell)$ value increases from top to bottom in figure 3(e) and other morphological parameters fixed are: fractal dimension $\left(D_{H}=1.4\right)$, strength of fractality $\left(\mu=15 \times 10^{-2}\right.$ (arbitrary units)) and upper length scale cut-off $(L=4 \mu \mathrm{m})$. In this plot we observe that the magnitude of imaginary component of impedance $\left(Z^{\prime \prime}(\omega)\right)$ decreases with the increase in lower cut-off length scale. Other qualitative responses are similar as we observed in figure 3(b).

In figure 3(c), we observe that the roughness can also be characterized by the strength of fractality (topothesy), i.e. linearly related to the mean square width (thickness) of the interface. Dependence of slope of intermediate region on $\mu$, indicate that even under limit, $\ell \rightarrow 0$ and $L \rightarrow \infty$ scaling exponent $(\beta)$ in (5) will be a function of $D_{H}$ and $\mu$ (an approximate power-law behaviour and dependency of dominant fractal morphological parameter shown in $\log -\log$ plot of magnitude of impedance with frequency in reference 44). This indicates the fact that the sole dependence of $\beta$ on $D_{H}$, as claimed in earlier work, is an incomplete characterization of $\beta$ with fractal morphological parameters. Here, the magnitude of imaginary component of impedance $\left(Z^{\prime}(\omega)\right)$ increases with increase in strength of fractality $(\mu)$. The linearity of Nyquist plots over a range of frequency implies an approximately identical functional dependence. This approximate functional dependence is power-law form in intermediate frequency regions.

Figure 3(f) shows the influence of strength of fractality $(\mu)$ over the impedance response of statistically corrugated fractal electrode. Strength of fractality increases from bottom to top curve while other surface morphological parameters are fixed. The value of strength of fractality varying here differs largely from the isotropic self-affine fractal surfaces to illustrate the similar qualitative response. From this plot we observed that the magnitude of imaginary component of impedance $\left(Z^{\prime \prime}(\omega)\right)$ increases with increase in strength of fractality for given value of real component of impedance $\left(Z^{\prime}(\omega)\right)$.

The qualitative description of response for both kinds of surfaces looks similar but they differ a lot in their detailed characteristics. These interesting and notable features which differentiate both kinds of surfaces can be analysed through the comparative study and dimensionality effect and can be seen in figure 4 . 


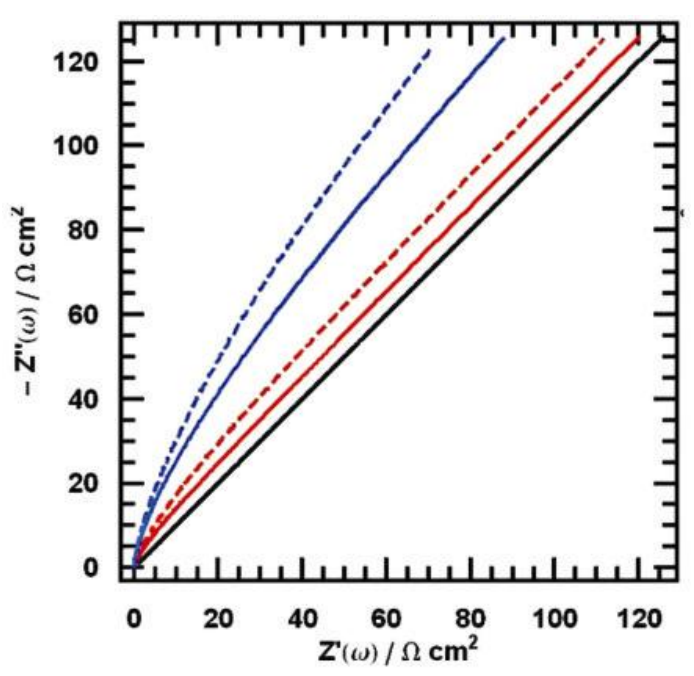

Figure 4. Comparison between isotropic and corrugated random fractals. Lowest solid line corresponds to planar Warburg response. Other lines correspond to isotropic fractals and dashed lines corresponding to corrugated fractals. The value of different parameters are (i) for isotropic, $D_{H}=2 \cdot 3,2 \cdot 4, \ell=40 \mathrm{~nm}, \mu=2 \times 10^{-6}$ (a.u.) and $L=4 \mu \mathrm{m}$, (ii) for corrugated $D_{H}=1 \cdot 3,1 \cdot 4, \ell=40 \mathrm{~nm}$, $\mu=15 \times 10^{-2}$ (a.u.) and $L=4 \mu \mathrm{m}$.

Figure 4 clearly differentiates between the corrugated and isotropic surface fractal response which is the effect of dimensionality of roughness. As we know, the degree of irregularity plays an important role in the electrochemical response of fractal electrode. Since, isotropic fractal surfaces show fluctuation independent of direction and have high roughness factor, while the corrugated surface shows fluctuation in one direction only, due to which it has comparative low roughness factor for same set of roughness parameters. From (8) and (11) one can say that the roughness contribution is directly proportional to roughness factor; that is why the impedance response for isotropic surface is high compared to corrugated surface for same value of roughness parameters and can be seen through figure 4 , where we increase the strength of fractality which is directly related to width of interface and roughness factor. In general, one can say that the magnitude of imaginary component of impedance $\left(Z^{\prime \prime}(\omega)\right)$ increases with increase of roughness and decrease in dimensionality. Roughness of the fractal surfaces increases with increase in fractal dimension, increase in strength of fractality and decrease in lower length cut-off. Therefore, one can easily say that fractal morphological parameters $\left(D_{H}, \ell, \mu\right)$ and dimensionality play an important role in the electrochemical impedance response in realistic fractals.

\section{Conclusion}

In this paper, we report theoretical results for the Nyquist impedance analysis for self-affine corrugated as well as self-affine isotropic fractal surfaces. We are interested to know the electrochemical response in term of admittance/impedance for surfaces with irregularities which are characterized as limited scales fractals. ${ }^{50}$ These disorders originate in the systems either from initial preparation/treatments (cleaning of surface) or their development in situ. The extent of deviation in response is dictated by the so called amplitude parameter, $h \propto \sqrt{\mu}$, of the profile as well as several other length scales in the problem. Here, we show that the admittance/ impedance of rough surfaces is not only characterized by fractal dimension $\left(D_{H}\right)$, but also on other surface morphological parameters i.e. $\ell$ and $\mu$. Nyquist impedance plots depend on three dominant morphological parameters of fractal, i.e. $D_{H}, \ell$ and $\mu$, this was not conceived in earlier work. The limited length scales fractal surface (corrugated as well as isotropic) model led to other important conclusions which are given as: (i) Anomalous diffusive behaviour in the intermediate frequency domain is due to fractal nature of surface on intermediate length scales. (ii) Our work clearly unravels the connection between the fractal dimension of roughness, the inner and outer cut-off length scales of roughness. (iii) Effect of dimensionality on electrochemical impedance is also analysed. Finally, one can say that our theory provides insight into the quantitative description of the role of roughness on the generalized Warburg impedance.

\section{Acknowledgements}

R K thanks University of Delhi for financial support (scheme to strengthen R\&D Doctoral Research Programme). R Kumar is grateful to the University Grants Commission (UGC), New Delhi for 'Research Fellowship in Science for Meritorious Students'.

\section{References}

1. Cottis S, Turgoose S and Newman R 2000 Corrosion testing made easy: Impedance and noise analysis (NACE international, Houston, Tex)

2. Yang L and Li Y 2005 Biosens. and Bioelectron. 20 1407 
3. Quanchao Z, Zuofeng C, Quanfeng D, Yanxia J, Ling H and Shigang S 2006 Chin. Sci. Bull. 511055

4. Urbain M, Rael S and Davat B 2007 Energetical modeling of lithium-ion batteries (Industry Application Conference, 42nd IAS Annual Meeting, IEEE)

5. Pfuch A, Heft A, Weidl R and Lang K 2006 Surf. Coat. Technol. 201189

6. Sarac A S, Sezgin S, Ates M and Turhan C M 2008 Surf. Coat. Technol. 2023997

7. Bevilaqua D, Acciari H A, Arena F A, Benedetti A V, Fugivara C S, Filho G T and Junior O G 2008 Miner. Eng.; doi:10.1016/j.mineng.2008.07.010

8. Yoon K H, Jang J H and Cho Y S 1998 J. Mater. Sci. Lett. 171755

9. Yaropolov A, Shleev S, Zaitseva E, Emnéus J, Marko-Varga G and Gorton L 2007 Bioelectrochemistry 70199

10. Bard A J and Faulkner L R 1980 Electrochemical methods: Fundamentals and application (New York: Wiley)

11. Lasia A 1999 Modern aspects of electrochemistry (eds) B E Conway, J O' M Bockris and R E White (New York: Kluwer Acad; Plenum) No. 32

12. Macdonald D D 2006 Electrochim. Acta 511376

13. De Gennes P G 1982 C.R. Acad. Sci. (Paris) Ser. II 2951061

14. Kopelman R 1986 J. Stat. Phys. 42 185; 1988 Science 2411620

15. Chaudhari A, Yan C-C S and Lee S-L 2002 Chem. Phys. Lett. 351341

16. Dewey T G 1994 Proc. Natl. Acad. Sci USA 9112101

17. Vandembroucq D, Boccaro A C and Roux S 1995 Europhys. Lett. 30209

18. Sapoval B 1996 Fractal electrodes, fractal membranes and fractal catalyst in fractals and disordered systems (eds) A Bunde and S Havlin (Heidelberg: Springer-Verlag)

19. Gutfraind R and Sapoval B 1993 J. Phys. I France 3 1801

20. Nyikos L and Pajkossy T 1986 Electrochim. Acta 31 1347

21. Pajkossy T and Nyikos L 1989 Electrochim. Acta 34 171

22. Nyikos L and Pajkossy T 1990 Electrochim. Acta 35 1567

23. Pajkossy T 1991 J. Electroanal. Chem. 3001

24. De Levie R 1990 J. Electroanal. Chem. 2811
25. Ramesh P and Sampath S 2003 Anal. Chem. 756949

26. Kant R and Rangarajan S K 2003 J. Electroanal. Chem. 552141

27. Kant R 1997 J. Phys. Chem. B101 3781

28. Kant R and Jha S K 2007 J. Phys. Chem. C111 14040

29. Jha S K, Sangal A and Kant R 2008 J. Electroanal. Chem. 615180

30. Go J-Y and Pyun S-I 2005 Electrochim. Acta 503479

31. Ocon P, Herrasti P, Vazquez L, Salvarezza R C, Vara J M and Arvia A J 1991 J. Electroanal. Chem. 319 101

32. Go J-Y and Pyun S-I 2007 J. Solid State Electrochem. 11323

33. Pajkossy T, Borosy A P, Imre A, Martemyanov S A, Schiller R and Nyikos L 1994 J. Electroanal. Chem. 36669

34. Kant R 1993 Phys. Rev. Lett. 704094

35. Kant R and Rangarajan S K 1994 J. Electroanal. Chem. 3681

36. Kant R and Rangarajan S K 1995 J. Electroanal. Chem. 396285

37. Kant $\mathrm{R}$ and Jha $\mathrm{S} \mathrm{K}$ Theory of partial diffusionlimited interfacial transfer/reaction on realistic fractals (Unpublished results)

38. Dassas Y and Duby P 1995 J. Electrochem. Soc. 142 4175

39. Imre A, Pajkossy T and Nyikos L 1992 Acta Metall. Mater. 401819

40. Palasantzas G 2005 Surf. Sci. 582151

41. Rangarajan S K 1969 J. Electroanal. Chem. 22 89

42. Bisquert J and Compte A J $2001 \mathrm{~J}$. Electroanal. Chem. 499112

43. Ball R and Blunt M 1988 J. Phys. A: Math. Gen. 21 197

44. Kant R, Kumar R and Yadav V K 2008 J. Phys. Chem. C112 4019

45. Warburg E 1899 Ann. Physik 67493

46. Maritan A, Stella A L and Toigo F 1989 Phys. Rev. B40 9269

47. Yordanov O I and Atanasov I S 2002 Euro Phys. J. B29 211

48. Niklasson G A et al 2000 Thin Solid Films 359203

49. Abramowitz M and Stegan A (eds) 1972 Handbook of mathematical functions (New York: Dover Publications Inc)

50. Feder J 1988 Fractals (New York: Plenum) 